ORIGINAL ARTICLE

\title{
Systemic mastocytosis with associated clonal haematological non-mast cell lineage diseases: a histopathological challenge
}

\author{
H-P Horny, K Sotlar, W R Sperr, P Valent
}

J Clin Pathol 2004;57:604-608. doi: 10.1136/jap.2003.014860

See end of article for authors' affiliations

.....................

Correspondence to: Professor H-P Horny, Institute of Pathology, University of Lübeck, Ratzeburger Allee 160, D-23538 Lübeck,

Germany; horny@patho. mu-luebeck.de

Accepted for publication 16 December 2003

\begin{abstract}
Aims: Although systemic mastocytosis (SM) with an associated clonal haematological non-mast cell lineage disease (SM-AHNMD) is a major subtype of SM, little is known about its frequency among myelogenous neoplasms, and mastocytosis in particular, or about AHNMD subtype frequencies.

Methods: Approximately 19500 routine bone marrow biopsies were evaluated. Immunostaining with antibodies against tryptase, KIT, and CD25 and molecular analysis for detection of C-KIT point mutations were performed in approximately 550/4100 myelogenous malignancies including mastocytosis, almost all subtypes of myelodysplastic syndrome (MDS), myelodysplastic/myeloproliferative syndrome (MDS/ MPD), MPD, and acute myeloid leukaemia (AML).

Results: SM was rare-it was diagnosed in only 64 bone marrows $(0.3 \%$ ) and made up $1.5 \%$ of myelogenous tumours. SM-AHNMD was the second most frequent subtype (20). SM-AHNMD was never included in the clinical differential diagnoses and was confirmed histologically in most cases only after appropriate immunostaining. The abnormal mast cell phenotype was confirmed by immunohistochemical demonstration of tryptase and CD25 coexpression. The following associated haematological neoplasms were found: MDS/MPS, AML, MPS, MDS, plasma cell myeloma, and unclassifiable myelogenous malignancy. C-KIT point mutations were detected in 16 of 20 cases.

Conclusions: SM-AHNMD can be diagnosed histologically in bone marrow trephines only after immunostaining with antibodies against tryptase, KIT, and CD25. Eighteen of 20 AHNMDs were of myeloid origin. C-KIT point mutations were present in 16 of 20 cases. The prognostic relevance of detecting SM associated with another haematological neoplasm remains unclear, but mast cell resistance to most cytoreductive agents is of major importance for treatment planning.
\end{abstract}

- How frequent is mastocytosis within the spectrum of haematological diseases on the basis of an evaluation of routinely processed bone marrow trephine specimens and how often is SM-AHNMD included in the clinical differential diagnosis?

- How frequent is SM-AHNMD within the spectrum of myelogenous neoplasms in general and SM in particular?

associated with cutaneous mastocytosis (urticaria pigmentosa); (b) aggressive SM, usually without skin involvement; (c) SM with an associated clonal haematological non-mast cell lineage disease (SM-AHNMD); and (d) mast cell leukaemia. ${ }^{1}$ Pure cutaneous mastocytosis and mast cell sarcoma are listed as separate, non-systemic entities. The only major diagnostic criterion for SM is the histological demonstration of compact tissue infiltrates, consisting of at least 10 to 15 mast cells. ${ }^{2}$ Recently, immunohistochemistry on routinely processed bone marrow biopsy specimens demonstrated that CD25 is expressed exclusively on mast cells of those cases with morphologically and molecular biologically confirmed mastocytosis, but not on mast cells in states of mast cell hyperplasia, enabling an abnormal or neoplastic phenotype of mast cells to be defined. ${ }^{3}$ CD25 expression on mast cells, prominent spindling of mast cells, and the demonstration of a point mutation of C-KIT (D816V) are three of the four minor criteria that are needed to establish a diagnosis of SM. Because a diagnosis of SM can be made when the major criterion and at least one of the minor criteria are fulfilled, the haematopathologist plays a crucial role in diagnosing or excluding mastocytosis in a given tissue specimen. Our present study was performed to answer the following questions:

- What is the frequency distribution of the associated haematological malignancies in SM-AHNMD?

- What is the frequency of point mutations of C-KIT in SMAHNMD?

\section{METHODS}

Approximately 19500 routinely processed bone marrow biopsy specimens were investigated between January 2000 and October 2003 at the institute of pathology (reference centre for haematopathology) of the University of Lübeck, Lübeck, Germany. The tissues were fixed in 5\% neutral formalin, mildly decalcified in edetic acid for six to eight hours, and embedded in paraffin wax. ${ }^{4}$ Conventional stains, such as Giemsa, Gömöri's silver impregnation, and naphthol AS-D chloroacetate esterase, were applied to all trephine specimens. In most myelogenous neoplasms (myelodysplastic and myeloproliferative syndromes and acute myeloid leukaemia), sections were immunostained using the avidinbiotin complex method with antibodies against various stem cell associated (CD34, CD38, and terminal deoxynucleotidyl

Abbreviations: $A H N M D$, associated clonal haematological non-mast cell lineage disease; AML, acute myeloid leukaemia; CMML, chronic myelomonocytic leukaemia; MDS, myelodysplastic syndrome; MPD, myeloproliferative syndrome; PCR, polymerase chain reaction; SM, systemic mastocytosis; WHO, World Health Organisation 
transferase), myelomonocytic (myeloperoxidase, lysozyme, CD15, and CD68), and mast cell related (tryptase, chymase, CD25, and CD117) antigens. ${ }^{5}$ Molecular biological studies were performed using peptide nucleic acid mediated polymerase chain reaction (PCR) clamping and Light Cycler hybridisation probes, as described previously. ${ }^{6}$ In selected cases, microdissected mast cells were investigated for the presence of C-KIT mutations, as also described previously. ${ }^{7}$ Diagnoses of haematological neoplasms including mastocytosis were established according to the WHO classification. The clinical records of the patients with SM-AHNMD were evaluated, in particular with respect to suspected mastocytosis.

\section{RESULTS}

Regarding the morphological evaluation of routinely processed bone marrow biopsy trephines, SM, irrespective of the subtype, comprised approximately $0.3 \%$ of all haematological diagnoses (approximately 19500 ), and nearly $1.5 \%$ of all myelogenous neoplasms (approximately 4100). Within the spectrum of mastocytosis, SM-AHNMD was the second most frequent type with a total of 20 cases (indolent SM including bone marrow mastocytosis and smouldering mastocytosis, 35; aggressive SM, seven; mast cell leukaemia, two). The clinical records of the patients with SM-AHNMD never included a differential diagnosis of mastocytosis. The age of these patients ranged from 45 to 78 years with a median of 65 years. The male to female ratio was nearly $2: 1$. In most of the cases ( 18 of 20 ), the clinical picture resembled that of a myeloproliferative or myelodysplastic syndrome; only two patients were recorded to have a monoclonal gammopathy of undetermined significance.

In most of the cases, compact mast cell infiltrates were obscured by the associated haematological neoplasm (AHNMD) when only haematoxylin and eosin or Giemsa stained sections were investigated. Meticulous analysis of the whole biopsy specimen using high power magnification sometimes revealed a few small foci consisting of spindle shaped cells with intermingled macrophages and eosinophils, and a pronounced increase in reticulin fibres. It was easier to detect such infiltrates within sheets of relatively monotonous blast cells than in the much more heterogeneous myelodysplastic or myeloproliferative syndromes. In almost all cases (19 of 20), the bone marrow was extremely hypercellular, with subtotal depletion of fat cells and widely effaced normal microarchitecture. Depending on the AHNMD, the morphological picture was dominated by sheets of blast cells (in cases of AML; $\mathrm{n}=4$ ), an increased atypical and left shifted granulocytopoiesis and erythrocytopoiesis (in MDS; $\mathrm{n}=2$ ), increased numbers of pleomorphic often clustered megakaryocytes (in MPS; $\mathrm{n}=3$ ), a diffuse increase in immature myeloid and monocytoid cells (in MDS/MPS, usually chronic myelomonocytic leukaemia of type CMMLl; $\mathrm{n}=7$ ), or focal accumulations of atypical plasma cells (in plasma cell myeloma; $n=2$ ). In two cases, the AHNMD could not be classified on the basis of the histopathological aspect alone, but was found to fit best into the category of a MDS/MPS. The only case of SM-AHNMD with hypocellular marrow was that of an associated plasma cell myeloma, with only slight infiltration of the bone marrow and secondary light chain associated (AL) amyloidosis. Table 1 lists the definitive diagnoses of the AHNMDs.

Immunohistochemically, the demonstration of at least one compact dense mast cell infiltrate was very easy when antitryptase staining was applied (figs 1 and 2). The accumulations of tryptase expressing mast cells were sharply demarcated from the surrounding bone marrow. In almost all cases, more than $25 \%$ of the mast cells exhibited a spindle shaped morphology. The mast cells coexpressed KIT (CD117)
Table 1 Systemic mastocytosis with an associated clonal haematological non-mast cell disease (SM-AHNMD): list of diagnoses of the AHNMDs and biological features of neoplastic cells

\begin{tabular}{|c|c|c|c|}
\hline AHNMD & $\begin{array}{l}\text { No. of } \\
\text { cases }\end{array}$ & C-KIT mutation (N) & CD25+ \\
\hline MDS: RARS & 1 & D816V (1) & $1 / 1$ \\
\hline MDS: RAEB 1 & 1 & D816V (1) & $1 / 1$ \\
\hline MDS/MPS (CMML) & 7 & $\begin{array}{l}\text { D816V (5) } \\
\text { D816Y (1) } \\
\text { wt (1) }\end{array}$ & $7 / 7$ \\
\hline MPS: PVR & 1 & wt (1) & $1 / 1$ \\
\hline MPS: CIM & 2 & D816V (2) & $2 / 2$ \\
\hline AML MI & 1 & No amplification & $1 / 1$ \\
\hline AML M2 & 2 & $\mathrm{D} 816 \mathrm{~V}(2)$ & $2 / 2$ \\
\hline AML M4 & 1 & wt (1) & $1 / 1$ \\
\hline Plasma cell myeloma & 2 & $\begin{array}{l}\text { D816V (1) } \\
\text { D816Y (1) }\end{array}$ & $2 / 2$ \\
\hline $\begin{array}{l}\text { NC (probably MDS/ } \\
\text { MPS) }\end{array}$ & 2 & D816V (2) & $2 / 2$ \\
\hline Total & 20 & 16 & 20 \\
\hline \multicolumn{4}{|c|}{$\begin{array}{l}\text { AML, acute myeloid leukaemia; CIM, chronic idiopathic myelofibrosis; } \\
\text { CMML, chronic myelomonocytic leukaemia; D816V, Asp816 } \rightarrow \text { Val; } \\
\text { D816Y, Asp816 } \rightarrow \text { Tyr; MDS/MPS, myelodysplastic/myeloproliferative } \\
\text { syndrome; NC, not classifiable; PVR, polycythaemia vera rubra; RAEB1 } \\
\text { refractory anaemia with excess of blasts (5-10\%); RARS, refractory } \\
\text { anaemia with ringed sideroblasts; wt, wild-type. }\end{array}$} \\
\hline
\end{tabular}

in a specific anular pattern, but were also positive for CD25, thus exhibiting an abnormal immunophenotype. Not only the lesional mast cells within the compact infiltrates, but also the loosely scattered mast cells, coexpressed tryptase and CD25. One exceptional case with an associated CMML showed only loosely scattered spindle shaped mast cells, sometimes aggregated into small perivascular clusters. Compact mast cell infiltrates according to the major diagnostic criterion of the WHO classification were not detected. Nevertheless, because three minor criteria ("spindling", "expression of CD25", and "D816V point mutation") could be demonstrated, a diagnosis of SM-AHNMD was established. It is of interest that one case of SM with an associated AML exhibiting the $\mathrm{t}(8 ; 21)$ translocation could not be diagnosed in the initial trephine biopsy. The second bone marrow biopsy removed after induction chemotherapy revealed disseminated compact infiltrates of atypical mast cells coexpressing tryptase and CD25 in a widely aplastic oedematous bone marrow. Re-evaluation of the initial tissue showed a slight increase of loosely scattered spindle shaped mast cells expressing tryptase and CD25, but compact infiltrates could not be detected. However, there was one paratrabecular small complex of about 10 mast cells reminiscent of a distorted compact infiltrate partly replaced by the AML blast cells. Because the point mutation D816V was detected in this initial trephine biopsy specimen, this case also fulfilled three minor diagnostic criteria, enabling the diagnosis of SM-AHNMD to be made. The plasma cell myelomas exhibited unusual features. One case was that of an extremely rare $\operatorname{IgM} / \kappa$ plasma cell myeloma, one case showed only slight infiltration of the bone marrow by neoplastic plasma cells of $\operatorname{IgG} / \lambda$ type associated with a generalised secondary AL amyloidosis, which involved the small bowel, leading to the initial presentation of an exudative enteropathy.

Molecular biological findings revealed the typical point mutation within the C-KIT protooncogene (D816V) in 14 of 20 cases, and uncommon point mutations (D816Y) in a further two cases associated with CMML and AMLM2. Three patients were found to exhibit the wild-type C-KIT alleles (SM associated with CMML, AMLM4, and polycythaemia vera) by peptide nucleic acid mediated PCR clamping. In one 


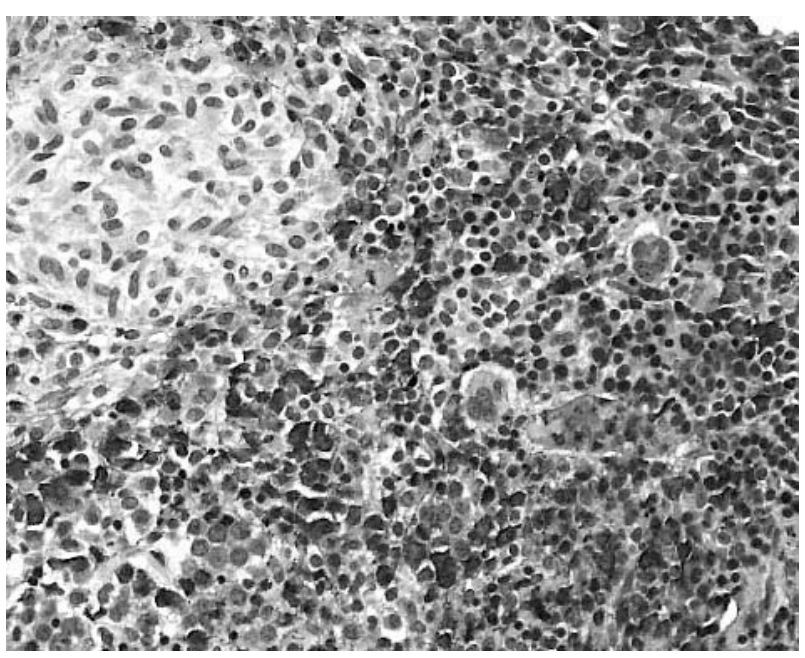

Figure 1 Systemic mastocytosis (SM) with an associated clonal haematological non-mast cell lineage disease: SM with an associated acute myeloid leukaemia. Chloroacetate esterase (CAE) staining shows an extremely hypercellular bone marrow with a monotonous picture, which is dominated by strongly positive atypical myeloid cells and blast cells. Note the periphery of a small CAE negative infiltrate at the upper left quadrant of the picture. Naphthol AS-D chloroacetate esterase stain.

of these three cases, microdissected mast cells were investigated but also exhibited the wild-type allele. In a final case, amplifiable DNA could not be extracted (association with AMLM1). With the exception of an associated AML with the $t(8 ; 21)$ translocation, other cytogenetic abnormalities of the AHNMDs were not recorded.

\section{DISCUSSION}

The main findings of this study can be summarised as follows:

- Among the four defined subtypes of SM, indolent SM was the most frequent $(\mathrm{n}=35)$, with SM-AHNMD $(\mathrm{n}=20)$ next in line, and therefore much more common than aggressive $\mathrm{SM}(\mathrm{n}=7)$ or the very rare true mast cell leukaemia $(n=2)$.

- Clinically, mastocytosis was never included in the differential diagnosis. Therefore, SM-AHNMD proved to be primarily a histological diagnosis that apparently depends on the experience of the haematopathologist, particularly his or her familiarity with mast cell proliferative disorders.

- The frequency distribution of the AHNMD shows a pronounced predominance of myelogenous neoplasms ( 18 of 20 ), in particular AML $(n=4)$ and MDS/MPS $(\mathrm{n}=7)$

- Sixteen of the 20 cases with SM-AHNMD showed a point mutation of the C-KIT protooncogene, mostly of the common type D816V (14 of 16).

- Mast cells of both the compact infiltrates and the diffuse (loosely scattered) compartments exhibited an abnormal immunophenotype, with coexpression of tryptase and CD25, which is never encountered in normal/reactive mast cells. These findings are of particular importance for the discrimination of mastocytosis from mast cell hyperplasia and for the diagnosis of indolent SM in patients with
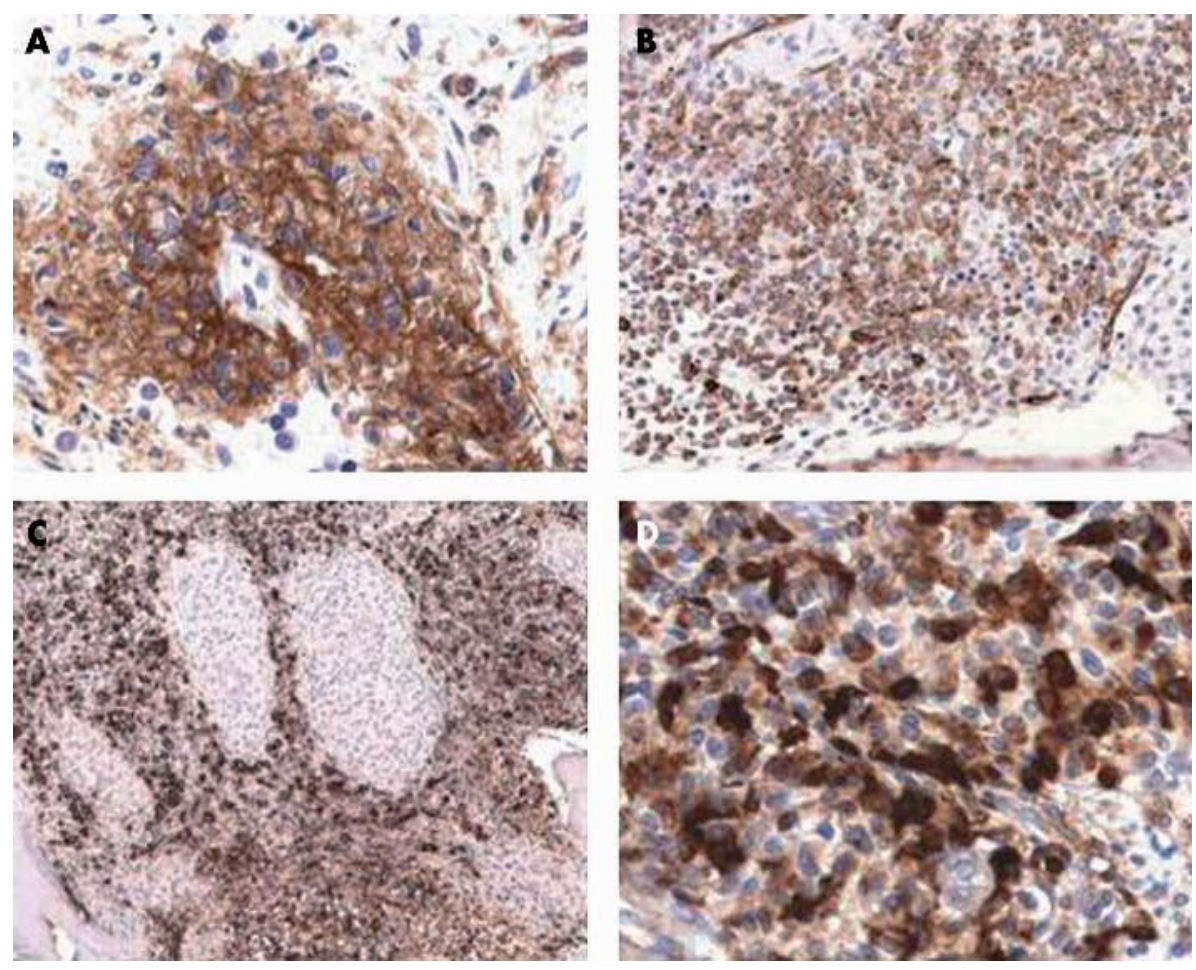

Figure 2 Systemic mastocytosis with an associated clonal haematological non-mast cell lineage disease (SM-AHNMD): SM with an associated acute myeloid leukaemia (SM-AML). (A) The mast cells coexpress CD25, underlining their neoplastic nature and confirming the diagnosis of SM-AMLM1. ABC method; anti-CD25. (B) There is coexpression of the stem cell associated antigen CD34 by the myeloblasts, whereas the mast cells are negative. The immunophenotype of the blast cells is consistent with the diagnosis of AMLM1. ABC method; Qbend10 (anti-CD34). (C) Immunostaining with antimyeloperoxidase reveals strong cytoplasmic reactivity of the myeloblasts, whereas the granulomatoid mast cell infiltrates remain unstained. Note the sharp demarcation of mast cell aggregates from the surrounding diffuse leukaemic infiltrate. Avidin-biotin complex (ABC) method; antimyeloperoxidase. (D) Higher magnification reveals strong cytoplasmic reactivity of the mast cells for antitryptase. $A B C$ method; $A A 1$ (antitryptase). 
cutaneous mastocytosis lacking compact mast cell infiltrates within the bone marrow.

Very little is known about the frequency distribution of mastocytosis within the spectrum of suspected haematological disorders, and about the subtypes of the associated haematological malignancies in SM-AHNMD. We found that mastocytosis is a very rare disorder, amounting to only $0.3 \%$ of diagnoses established on bone marrow biopsies (nearly 19500 ) evaluated in a reference centre for haematopathology (institute of pathology, University of Lübeck, Lübeck, Germany) within a period of about three years. Among the myelogenous tumours (approximately 4100), mastocytosis made up about $1.5 \%$ of all cases and thus belonged to the group of rare neoplasms. SM-AHNMD is a novel entity defined in the recent WHO classification of malignant tumours of the haemopoietic tissues and comprises all cases of SM with an associated non-mast cell lineage clonal haematological disease. ${ }^{1}$ As expected, myelogenous malignancies made up most of the AHNMDs (18 of 20), with a predominance of MDS/MPS $(n=7)$ and AML $(n=4)$, the former including a spectrum of myelogenous tumours, in particular CMML, which were defined in the recent WHO classification of malignant haemopoietic tumours. ${ }^{1}$

Our findings on the frequency distribution of AHNMDs are comparable to published data, which also reported a preponderance of myelogenous malignancies compared with overt lymphoproliferative disorders. ${ }^{89}$ The total number of published cases of SM-AML amounts to nearly 30 and covers the whole spectrum of subtypes according to the FAB classification, with a peak for AML types M2 and M5. ${ }^{89}$ Moreover, most subtypes of chronic myeloproliferative disorders have been described as AHNMDs, including essential thrombocythaemia. ${ }^{10}$ However, to the best of our knowledge, an association of SM with Philadelphia chromosome positive chronic myeloid leukaemia has not been reported. ${ }^{9}$ According to all published data, the most frequent myelogenous neoplasm associated with SM is CMML, which is included in the group of MDS/MPS. ${ }^{7811}$

According to our experience, the incidence of a diagnosis of SM-AHNMD should increase if appropriate immunostaining using antibodies against the diagnostically relevant mast cell related markers, such as tryptase (enabling the detection of even very small compact mast cell infiltrates) and CD25 (defining an abnormal immunophenotype of mast cells seen only in neoplastic states), were applied routinely in all bone marrow trephines containing infiltrates of MDS, AML, and CMML (MDS/MPS). Although tryptase cannot be regarded as a specific marker for mast cells (atypical basophils in chronic myeloid leukaemia and myeloblasts in AML have also been shown to express this protease) and CD25 (anti-interleukin 2 receptor) is not a specific marker for a particular haemopoietic cell (although it is expressed on basophils and a subset of $\mathrm{T}$ cells), it should be emphasised that cells coexpressing tryptase and CD25 in the setting of a haematological disease are probably abnormal mast cells and therefore can be regarded as a diagnostic clue for mastocytosis because basophils usually do not contain amounts of tryptase detectable by immunohistochemistry. ${ }^{12}{ }^{13}$

Diffuse dense blast cell infiltrates might obscure or even distort pre-existing compact mast cell infiltrates, a phenomenon we recently found in a patient with AMLM2 and $t(8 ; 21)$, in whom compact mast cell infiltrates were found only in the second bone marrow biopsy removed after induction chemotherapy with pronounced aplasia of blast cells and haemopoiesis. This case is described in detail in a recent publication. ${ }^{14}$

Regarding the low incidence of lymphoproliferative disorders within the spectrum of AHNMDs, we could add two patients with unusual types of plasma cell dyscrasia to the current literature. ${ }^{15}$ One patient had a plasma cell myeloma of $\operatorname{IgM} / \kappa$ subtype and the other a plasma cell myeloma of $\operatorname{IgG} / \lambda$ subtype, with associated secondary generalised amyloidosis. We are aware of only one other published case of SM plasma cell myeloma. ${ }^{16}$

With regard to the pathogenesis of mastocytosis, the detection of activating point mutations of C-KIT in a large proportion of patients with mastocytosis strongly indicates that most (all?) cases of mastocytosis, in particular the systemic or leukaemic variants, are clonal haematological neoplasms, obviously of bone marrow origin. According to our own largely unpublished observations, C-KIT mutations can be detected in about $80 \%$ of all patients with SM, especially when more sensitive molecular methods such as peptide nucleic acid mediated PCR clamping or nested PCR on pooled microdissected single mast cells are performed on routinely processed bone marrow biopsies. ${ }^{6}{ }^{17}$ We found point mutations of C-KIT in 16 of 20 of the bone marrow specimens containing tissue infiltrates of SM-AHNMD. Most (14 of 16) of these point mutations belonged to the so called common type, D816V, a finding that agrees with recently published data. ${ }^{17}$ The uncommon point mutation D816Y was detected twice. However, the point mutation recently described by Pullarkat et al in three of five (Asp816His) SM-AHNMDs was not found in our patients. ${ }^{18}$ It also seems that the variability of C-KIT mutations in bone marrow infiltrates of mastocytosis is very much lower than in cutaneous mastocytosis. ${ }^{6}$

\section{"Cells coexpressing tryptase and CD25 in the setting of a haematological disease are probably abnormal mast cells and therefore can be regarded as a diagnostic clue for mastocytosis"}

In a small proportion of the AHNMDs, other clonal markers were detected-for example, the 8;21 translocation in one of our patients with SM-AMLM2 and monoclonal gammopathies or paraproteinaemias $(\mathrm{IgM} / \kappa$ and $\operatorname{IgG} / \lambda$, respectively) in the two patients with SM plasma cell myeloma. ${ }^{14}{ }^{15}$ One of these patients presented clinically with a secondary intestinal amyloidosis, and the bone marrow biopsy was then performed to assess or exclude plasma cell myeloma. ${ }^{15}$ The detection of SM was unexpected in this patient. Such findings clearly raise the question of a possible pathophysiological relation between SM and the AHNMD. The most likely answer is that both haematological diseases evolve from an early uncommitted haemopoietic progenitor cell as a primary monoclonal disease, with further evolution into phenotypically different subclones. ${ }^{8}$ This hypothesis is the most likely in the most common forms of SM-AHNMD, namely those with associated myelogenous neoplasms. However, there is the possibility of the coincidental development of two distinct clonal haematological tumours. ${ }^{8}$ This concept would fit for the rare occurrence of SM associated with lymphoproliferative disorders/plasma cell dyscrasias. Interestingly, monoclonal gammopathy of unclear significance is much more common in patients with mastocytosis than overt B cell neoplasms/plasma cell myelomas and, moreover, point mutations of C-KIT have been detected in circulating B cells. ${ }^{19} 20$

Finally, is there any clinical relevance for the coincidental histomorphological detection of SM in the bone marrow of patients within the setting of another haematological malignancy? The clinical relevance of such histological, mostly unexpected, findings is largely unknown. It must be stated that as far as we know at this time, the "SM" in SMAHNMD should be treated like pure SM and the "AHNMD" should usually be treated in the same manner as pure 


\section{Take home messages}

- Systemic mastocytosis (SM) with an associated clonal haematological non-mast cell lineage disease SMAHNMD can be diagnosed histologically in bone marrow trephines only after immunostaining with antibodies against tryptase, KIT, and CD25

- Most AHNMDs are of myeloid origin and C-KIT point mutations were present in 18 of the 20 cases

- The prognostic relevance of detecting SM associated with another haematological neoplasm remains unclear, but mast cell resistance to most cytoreductive agents is of major importance for treatment planning

AHNMD. ${ }^{21}$ However, the resistance of neoplastic mast cells to cytoreductive drugs has to be borne in mind. In this regard, it is also noteworthy that imatinib is unlikely to be an effective agent in such patients because the D816V mutation of C-KIT converts KIT to an imatinib resistant kinase receptor. ${ }^{20}$ This fact is underlined by our findings in a patient with AML type M2 with $\mathrm{t}(8 ; 21)$ in whom treatment resulted in long standing complete haematological and cytogenetic remission of the AML but the disclosure and persistence of SM. ${ }^{14}$ In addition, a patient with AML associated with mast cell leukaemia was reported to achieve complete molecular remission of the AML soon after induction chemotherapy, whereas the mastocytosis persisted after standard chemotherapy and allogeneic stem cell transplantation. ${ }^{22}$

To summarise, SM-AHNMD is a primarily morphological diagnosis based on a thorough investigation of bone marrow trephine specimens including tryptase and CD25 immunohistochemistry. The most frequent AHNMDs are myelogenous neoplasms belonging to the group of myelodysplastic/ myeloproliferative syndromes (CMML) and acute myeloid leukaemias.

\section{Authors' affiliations}

H-P Horny, Institute of Pathology, University of Lübeck, D-23538 Lübeck, Germany

K Sotlar, Institute of Pathology, University of Tübingen, D-72076

Tübingen, Germany

W R Sperr, P Valent, University of Vienna, AKH, Department of Internal Medicine/Division of Haematology and Haemostaseology, A-1090 Vienna, Austria

\section{REFERENCES}

1 Valent P, Horny H-P, Li CY, et al. Mastocytosis. In: Tumours of haematopoietic and lymphoid tissues (WHO). Lyon, France: IARC Press, 2001:291-302.

2 Valent $\mathrm{P}$, Horny H-P, Escribano L, et al. Diagnostic criteria and classification of mastocytosis: a consensus proposal. Leuk Res 2001;25:603-25.
3 Horny H-P, Lange K, Sotlar K, Valent P. Increase of bone marrow lymphocytes in systemic mastocytosis: reactive lymphocytosis or malignant lymphoma? Immunohistochemical and molecular findings on routinely processed bone marrow biopsy specimens. J Clin Pathol 2003;56:575-8.

4 Horny H-P, Kaiserling E. Lymphoid cells and tissue mast cells of bone marrow lesions in systemic mastocytosis: a histological and immunohistological study. Br J Haematol 1988;69:449-55.

5 Hsu SM, Raine L, Fanger R. Use of avidin-biotin-peroxidase complex $(A B C)$ in immunoperoxidase techniques: a comparison between $A B C$ and unlabeled antibody (PAP) procedures. J Histochem Cytochem 1981;29:577-80

6 Sotlar K, Escribano L, Landt O, et al. One-step detection of C-KIT point mutations using PNA-mediated PCR-clamping and hybridization probes. Am J Pathol 2003;162:737-46.

7 Sotlar K, Fridrich C, Mall A, et al. Detection of C-KIT point mutation Asp-816 $\rightarrow \mathrm{Val}$ in microdissected pooled single mast cells and leukemic cells in a patient with systemic mastocytosis and concomitant chronic myelomonocytic leukemia. Leuk Res 2002;26:979-84

8 Sperr WR, Horny H-P, Lechner K, et al. Clinical and biologic diversity of leukemias occurring in patients with mastocytosis. Leuk Lymphoma 2000;37:473-86.

9 Sperr WR, Horny H-P, Valent P. Spectrum of associated clonal hematologic non-mast cell lineage disorders occurring in patients with systemic mastocytosis. Int Arch Allergy Immunol 2002;127:140-2.

10 LeTourneau A, Gaulard P, Dàgay MF, et al. Primary thrombocythaemia associated with systemic mastocytosis: a report of five cases. $\mathrm{Br} J$ Haematol 1991;79:84-9.

11 Travis WD, Li CY, Bergstralh EJ. Solid and hematologic malignancies in 60 patients with systemic mast cell disease. Arch Pathol Lab Med $1989 ; 113: 365-8$.

12 Samorapoompichit $\mathbf{P}$, Kiener HP, Schernthaner GH, et al. Detection of tryptase in cytoplasmic granules of basophils in patients with chronic myeloid leukaemia and other myeloid neoplasms. Blood $2001 ; 98: 2580-3$

13 Sperr WR, Hauswirth AW, Valent P. Tryptase as a novel biochemical marker of acute myeloid leukaemia. Leuk Lymphoma 2002:43:2257-61.

14 Bernd H-W, Sotlar K, Lorenzen J, et al. Acute myeloid leukaemia (AML) with $t(8 ; 21)$ associated with "occult" mastocytosis (SM-AHNMD). Report of an unusual case and review of the literature. J Clin Pathol 2004:57:324-8.

15 Stellmacher F, Sotlar K, Balleisen L, et al. Bone marrow mastocytosis associated with IgM kappa plasma cell myeloma. Leuk Lymphoma 2004;45:801-5.

16 Hagen W, Schwarzmeier J, Walchshofer S, et al. A case of bone marrow mastocytosis associated with multiple myeloma. Ann Hematol 1998;76: 167-74

17 Sotlar K, Marafioti T, Griesser H, et al. Detection of C-KIT mutation Asp-816 to $\mathrm{Val}$ in microdissected bone marrow infiltrates in a case of systemic mastocytosis associated with chronic myelomonocytic leukaemia. Mol Pathol 2000;53:188-93.

18 Pullarkat VA, Bueso-Ramos C, Lai R, et al. Systemic mastocytosis with associated clonal hematological non-mast-cell lineage disease: analysis of clinicopathologic features and activating c-kit mutations. Am J Hematol 2003;73:12-17.

19 Meggs WJ, Frieri M, Costello R, et al. Oligoclonal immunoglobulins in mastocytosis. Ann Int Med 1985;103:894-5.

20 Akin C, Kirshenbaum AS, Semere T, et al. Analysis of the surface expression of c-kit and occurrence of the c-kit Asp816Val activating mutation in T cells, B cells, and myelomonocytic cells in patients with mastocytosis. Exp Hematol 2000;28:140-7.

21 Valent $\mathbf{P}$, Akin C, Sperr WR, et al. Diagnosis and treatment of systemic mastocytosis: state of the art. Br J Haematol 2003;122: 695-717

22 Chen TY, Chen JS, Huang WT, et al. Rapid engraftment of mast cells of donor origin in a case of acute myeloid leukaemia with mast cell leukaemia after allogeneic stem cell transplantation. Bone Marrow Transplant 2003;32:111-14 